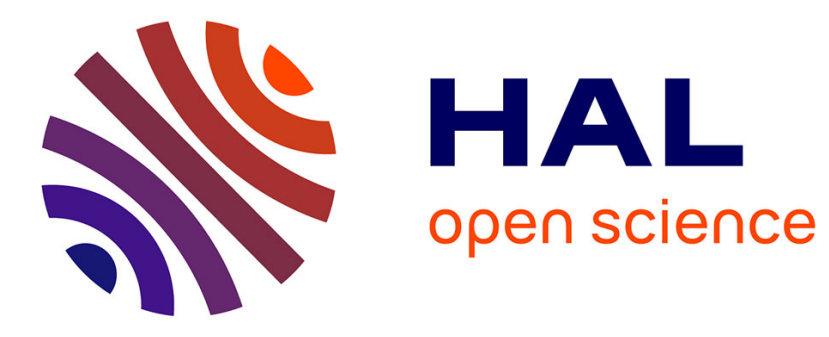

\title{
Automatic Streamization of Image Processing Applications
}

Pierre Guillou, Fabien Coelho, François Irigoin

\section{To cite this version:}

Pierre Guillou, Fabien Coelho, François Irigoin. Automatic Streamization of Image Processing Applications. The 27th International Workshop on Languages and Compilers for Parallel Computing, Parasol, Sep 2014, Hillsboro, United States. pp 224-238, 10.1007/978-3-319-17473-0_15 . hal-01096735

\section{HAL Id: hal-01096735 \\ https: / hal-mines-paristech.archives-ouvertes.fr/hal-01096735}

Submitted on 19 Dec 2014

HAL is a multi-disciplinary open access archive for the deposit and dissemination of scientific research documents, whether they are published or not. The documents may come from teaching and research institutions in France or abroad, or from public or private research centers.
L'archive ouverte pluridisciplinaire HAL, est destinée au dépôt et à la diffusion de documents scientifiques de niveau recherche, publiés ou non, émanant des établissements d'enseignement et de recherche français ou étrangers, des laboratoires publics ou privés. 


\title{
Automatic Streamization of Image Processing Applications
}

\author{
Pierre Guillou, Fabien Coelho, and François Irigoin \\ firstname. lastname@mines-paristech.fr
}

MINES ParisTech, PSL Research University

\begin{abstract}
New many-core architectures such as the Kalray MPPA-256 provide energy-efficiency and high performance for embedded systems. However, to take advantage of these opportunities, careful manual optimizations are required. We investigate the automatic streamization of image processing applications, implemented in $\mathrm{C}$ on top of a dedicated API, onto this target accessed through the $\Sigma \mathrm{C}$ dataflow language. We discuss compiler and runtime design choices and their impact on performance. Our compilation techniques are implemented as source-to-source transformations in the PIPS open-source compilation framework. Experiments show lowest energy consumption on the Kalray MPPA target compared to other hardware targets for a range of 8 test applications.
\end{abstract}

\section{Introduction}

As predicted by Moore's law, billions of transistors can be integrated today into a single chip, enabling multi-core or even many-core architectures, with hundreds of cores on a chip. The low energy consumption Kalray MPPA-256 processor [17], released in 2013, offers 256 computing VLIW-cores for $10 \mathrm{~W}$. Task and/or data parallel approaches can be used to take advantage of such parallel processing power for a given application domain.

We show how to use this innovative hardware to run image processing applications in embedded systems such as video cameras. In order to enable fast timeto-market developments of new products, applications must be ported quickly and run efficiently on these targets, a daunting task when done manually. To alleviate this issue, we have built a compiler chain to automatically map an image processing application developed on top of a dedicated software interface, FREIA [14], considered as a domain specific language, onto the MPPA processor using the $\Sigma \mathrm{C}$ dataflow language and runtime. Images are streamed line-by-line into a task graph whose vertices are image operators.

Streaming languages [31] have been studied for a long time, and have recently received more attention [28] for exposing pipelining, data parallelism and task paralellism, as well as hiding memory management. The Kahn Process Networks [22] are one of the first streaming model, relying on FIFOs for interprocess communication. As subclasses of this model, Synchronous DataFlow [25,26] languages are statically determined in order to avoid deadlocks and to ensure safety. 
Common SDF languages include LUSTRE [20], Signal [24] or StreamIt[1]. The latter shares some ground concepts with the $\Sigma \mathrm{C}$ dataflow language such as decomposing programs in a graph of basic interconnected units which consume and produce data or focusing on easing programming onto multi-core and manycore targets. In particular, some optimizations of StreamIt applications (operator replication to enable data parallelism, operator fusion to reduce communication overheads [18]) are close to those presented in this paper. Other projects such as DAGuE [9] or FlumeJava [10] propose frameworks for managing and optimizing tasks targetting current multi-core architectures.

Optimization of image processing applications on massively parallel architectures have been the subject of multiple studies. Ragan-Kelley [29] proposes an image processing Domain Specific Language and an associated compiler for optimizing parallelism onto standard CPUs or GPUs. Clienti [12] presents several dataflow architectures for specific image analysis applications. Stencil operators are the most limitating operators in our implementation. Several alternative techniques for optimizing this class of operators have been proposed $[16,6]$.

This paper focusses on the design of a compiler and a runtime using the MPPA chip as an accelerator, including: (1) domain specific transformations on the input code to expose larger image expressions; (2) code generation for a dataflow language; (3) automatic operator aggregation to achieve a better task balance; (4) a small runtime to provide streaming image operators; (5) dataparallel agents for slower operators. We also demonstrate the effectiveness of our approach with a source-to-source implementation in PIPS [21,15] by reporting the time and energy consumption on a sample of image applications.

We first describe the overall compilation chain in Section 2, then in Section 3 we focus on our hardware and software targets. Section 4 presents our key contributions about the compiler and runtime designs. Section 5 reports our time and energy performance results.

\section{Compilation Chain Overview}

The starting point of our compilation chain (Figure 1) is an image processing application, built on top of the FREIA C language API. This provides a 2D image type and dozens of functions to perform basic image operations (arithmetics, logical, morphological, reductions), as well as composed operators which combine several basic ones. Typical applications locate text in an image, smooth visible blocks from JPEG decoding, or detect movements in an infra-red surveillance video. An example of FREIA code is shown in Figure 3. Our test case applications typically include up to hundreds of basic image operations, with 42 as a median. These operations are grouped in few (1-3, up to 8) independent static image expressions that can be accelerated, stuck together with control code.

The ANR FREIA [7] project developed a source-to-source compilation chain [14] from such inputs to various hardware and software targets: the SPoC image processing FPGA accelerator [13], the TERAPIX 128 processing elements SIMD array FPGA accelerator [8], and multi-cores and GPUs using OpenCL [23]. This 


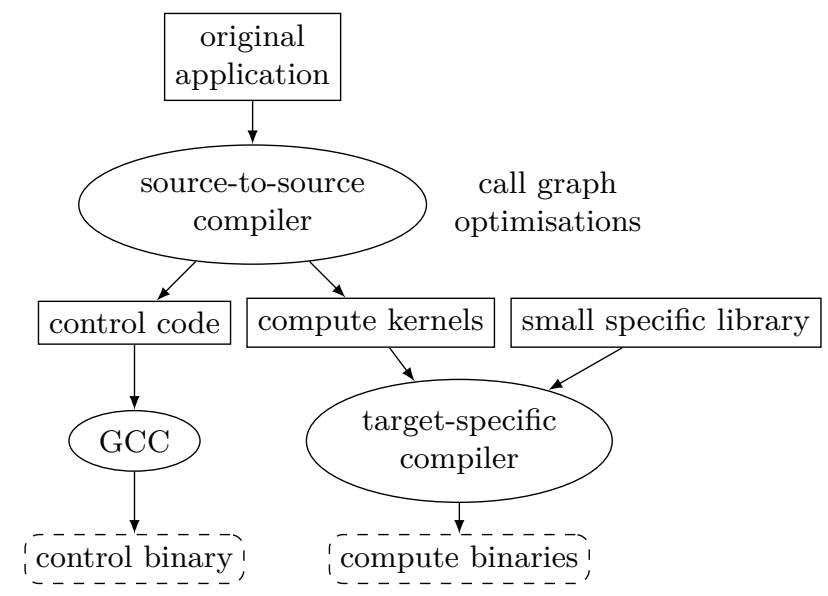

Fig. 1. Overview of our compilation chain

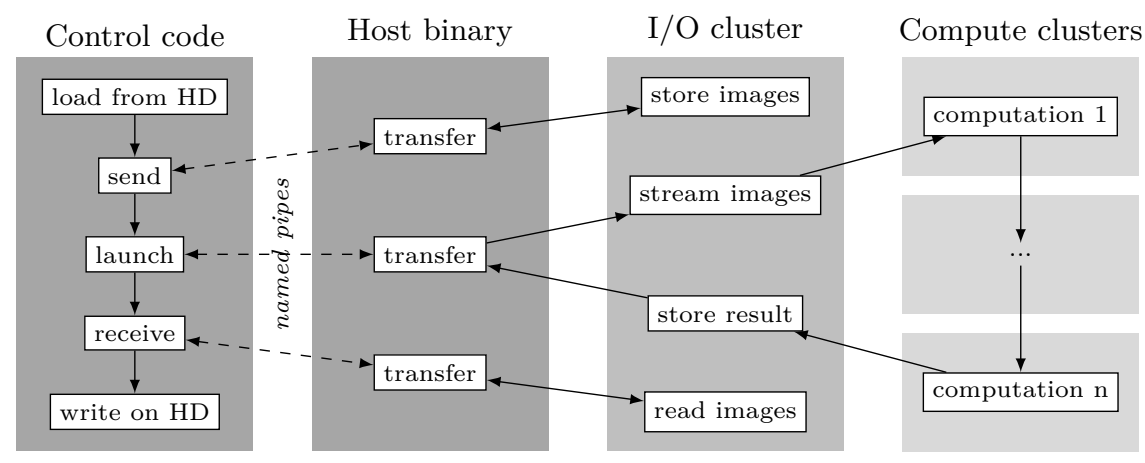

Fig. 2. Summary of our runtime environment

new development adds Kalray's MPPA-256 chip as a target hardware by providing a new code generation phase and its corresponding runtime.

The FREIA compiler chain is made of three phases. The first two phases are generic, and the last one is the target specific code generation. Phase 1 builds sequences of basic image operations, out of which large image expressions can be extracted. For this purpose, inlining of composed and user operators, partial evaluation, loop unrolling, code simplifications and dead code suppression are performed. An important preliminary transformation for a dataflow hardware target such as SPoC and MPPA is while-loop unrolling. Phase 2 extracts and optimizes image expressions, as a directed acyclic graph (DAG) of basic image operations. Optimizations include detecting common subexpressions, removing unused image computations, and propagating copies forwards and backwards.

The target execution model depicted in Figure 2 uses the parallel hardware as an accelerator for heavy image computation, while the host processor runs 


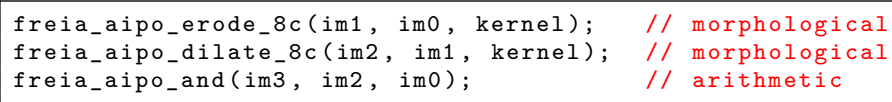

Fig. 3. Example FREIA code with a sequence of 3 operations

the control code and I/Os. The runtime environment includes functions for manipulating images such as allocate, receive, emit, and the various operators. The accelerated version has to manage the transfers between the host and the device used for operator computations. For our MPPA target, this is achieved by using named pipes to send images to agents on the host. Theses images are first streamed to the device for computation, then streamed back on a host agent, then back to the main program.

\section{Hardware and Software Target}

We are targetting the Kalray MPPA-256 many-core architecture through the $\Sigma \mathrm{C}$ dataflow language, compiler and runtime, which allows us to build a runtime for streaming image operators that can be connected to process large expressions.

\subsection{MPPA-256 Architecture}

Kalray MPPA-256 [17] is a high-efficiency $28 \mathrm{~nm}$ System-on-Chip featuring 256 compute cores providing 500 GOPs with a typical power consumption of $10 \mathrm{~W}$. Competing MPPA architectures include the Tilera TILEPro64 [3], the Adapteva Epiphany [4], or the TSAR Project [2]. This massively parallel processor aims at a wide range of embedded applications and boasts a fast time-to-market for complex systems.

Figure 4 shows MPPA-256's computes cores divided into sixteen compute clusters. Each one of these clusters includes a $2 \mathrm{MB}$ non-coherent shared L2 cache. The compute cores are 32 bits multithreaded Very Long Instruction Word cores, thus offering instruction parallelism. SIMD instructions operating on pairs of fixed-point or floating-point instructions are also supported. Every compute cluster also runs a minimalistic real-time Operating System (NodeOS) on a separate and dedicated core. This OS manages the 16 compute cores of one cluster by executing multithreaded binary programs onto them. The compute clusters communicate with each other through a high-speed toroidal Network-on-Chip.

The MPPA-256 chip also includes four additional clusters for managing external communications. These input/output clusters provide several interfaces, such as PCI-Express for connecting to a host machine, DDR interface to access local RAM, Ethernet or Interlaken for directly connecting several chips together.

For our work, the MPPA-256 served as hardware accelerator. The chip was placed aside a 4 GB dedicated RAM onto a PCI-Express extension card. This card was then accessed through the PCI-Express bus of a typical computer workstation. 


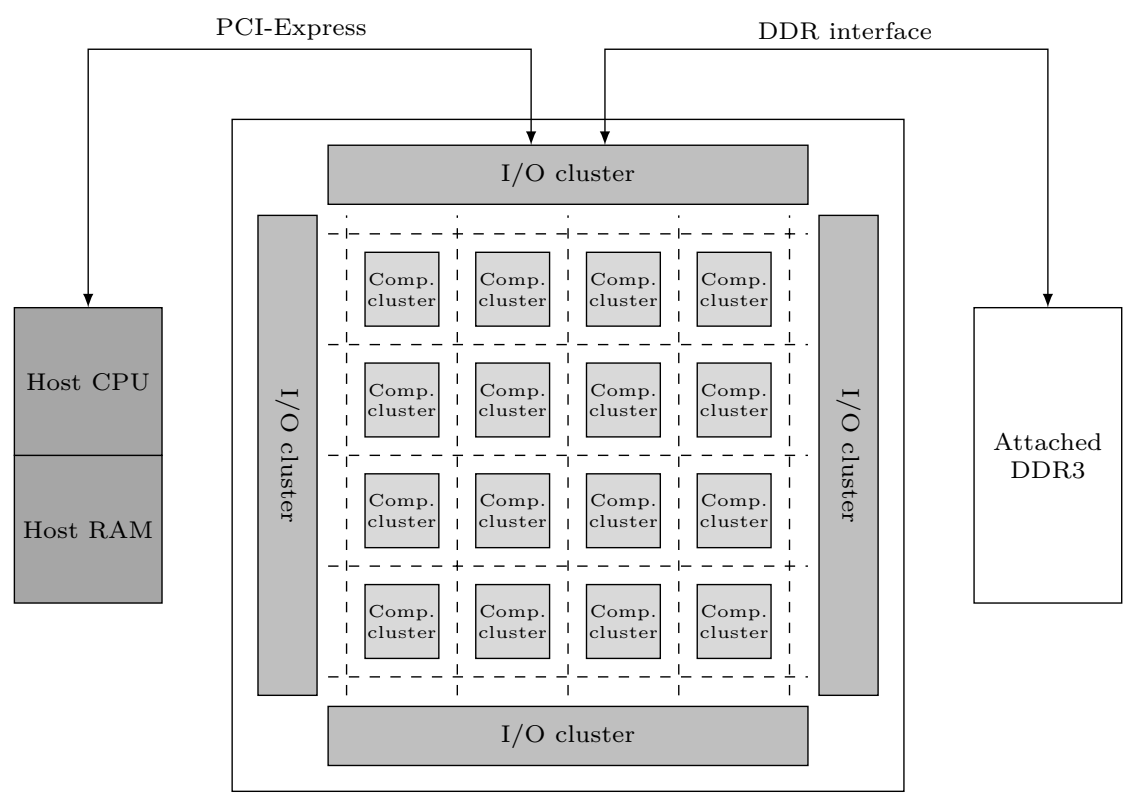

Fig. 4. The MPPA-256 chip and its environment

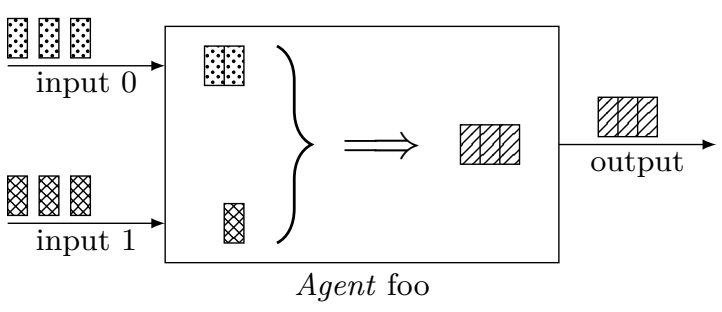

Fig. 5. A $\Sigma$ C agent with two inputs and one output (see code in Figure 6)

\section{$3.2 \quad$ The $\Sigma \mathrm{C}$ programming language}

To ease the programming on their manycore chip [5], Kalray offers the classic parallel libraries PThread and OpenMP [27] as well as a specific dataflow programming language called $\Sigma \mathrm{C}$ [19]. $\Sigma \mathrm{C}$ is a superset of the $\mathrm{C}$ which provides a dataflow programming model and relies on the concept of agents, which are basic compute blocks similar to Kahn Processes [22]. These blocks receive and consume a fixed amount of data from input channels, and produce data on their output channels. The agent represented in Figure 5 has two input channels and one output channel. When two pieces of data are available on the first input channel and one on the second, the agent produces three pieces of data on its sole output. Corresponding $\Sigma \mathrm{C}$ code is shown in Figure 6. 


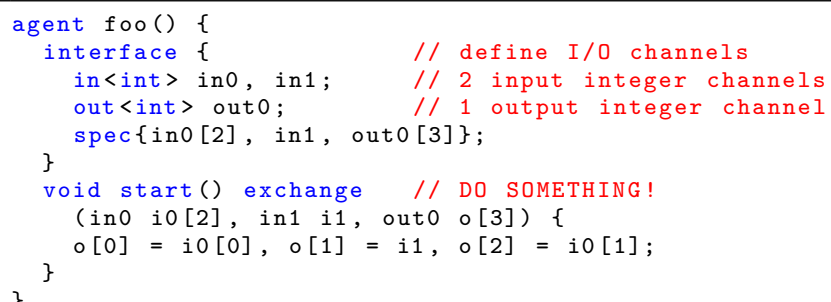

Fig. 6. $\Sigma \mathrm{C}$ example: a basic agent merging two integer streams (see Figure 5)

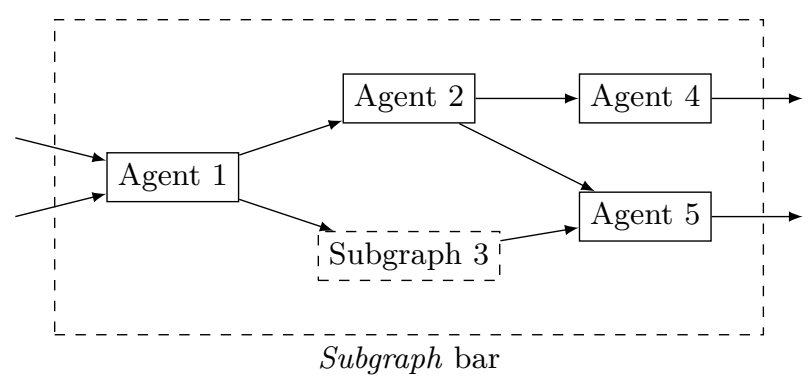

Fig. 7. A $\Sigma$ C subgraph composed of 4 agents and one subgraph (see code in Figure 8)

This model has two consequences. First, the scheduling of agents on available cores and the inter-agent buffer allocation requires the $\Sigma \mathrm{C}$ compiler to know the number of input/output channels of an agent, and the number of data items processed. This implies that image sizes must be known at compile time. Then, when several independent graphs are mapped, the compiler assumes that these graphs may be active at the same time, thus the mapping reserves nonoverlapping memory and cores for the tasks. If only one graph at a time is really active, resources can be under-used.

The performance model implies that tasks must provide significant computations in order to amortize communication costs. In particular, communication times include a constant overhead, which must be amortized with significant data volumes. However, as memory is scarse, it is best to require small intertask buffers: a trade-off must be made. Another key point of the static dataflow model is that the slowest task in the graph determines the overall performance. Therefore, elementary tasks must be as fast and as balanced as possible.

Agents can be connected to each other in order to compose a $\Sigma \mathrm{C}$ subgraph which can recursively compose upper-level subgraphs. A subgraph representation and the corresponding $\Sigma \mathrm{C}$ code are respectively showed in Figure 7 and Figure 8. The top-level subgraph is called root and corresponds to the classic $\mathrm{C}$ main function. A $\Sigma$ C agent can be executed either by one of MPPA-256's compute cores or by a core of an input/output cluster. Agents can also be executed by the processor of the host machine, therefore providing access to a hard drive. 


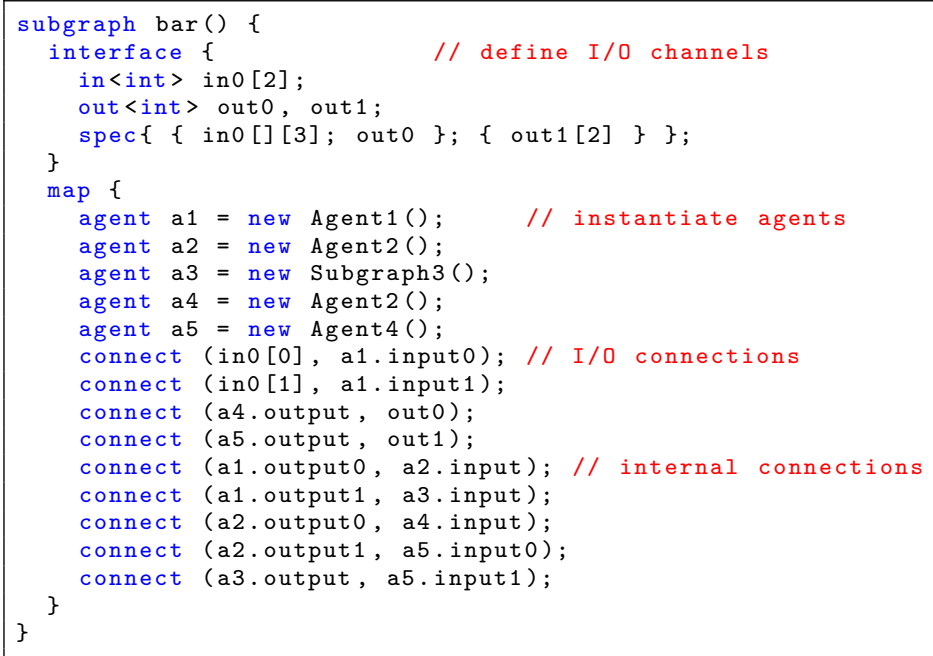

Fig. 8. $\Sigma$ C example: a basic subgraph (see graph in Figure 7)

Kalray provides also a $\Sigma \mathrm{C}$ compiler for MPPA-256, which handles the mapping of $\Sigma \mathrm{C}$ agents on the compute cores of their chip.

The $\Sigma \mathrm{C}$ programming language provides an effective way to take advantage of the MPPA-256, and serves as the main target language for demonstrating our automatic streamization compiler and runtime.

\section{Compiler and Runtime Design}

A DAG produced by our compilation chain has a similar structure to streaming programs. Indeed, image analysis operators can be directly transposed as $\Sigma \mathrm{C}$ agents, and DAGs as $\Sigma$ C subgraphs.

\section{1 $\Sigma$ C Image Processing Library}

Aside from our compilation chain, we developed a $\Sigma \mathrm{C}$ library of elementary image analysis operators. Each operator is implemented as one $\Sigma \mathrm{C}$ agent, such as: (1) arithmetic operators performing elementary operations onto pixels of input images; (2) morphological operators [30], which are the more compute intensive operators; (3) reduction operators returning a scalar value.

The $2 \mathrm{MB}$ per compute cluster memory limit implies that our $\Sigma \mathrm{C}$ agents cannot operate on a whole image. Since the transition between two states of an agent is rather slow, we cannot afford to operate on one pixel at a time. Thus our agents process images line by line. Measuring per pixel execution time of several applications for several input image sizes (see Figure 9) reveals that larger lines are computed more efficiently than shorter ones, as communication times are 


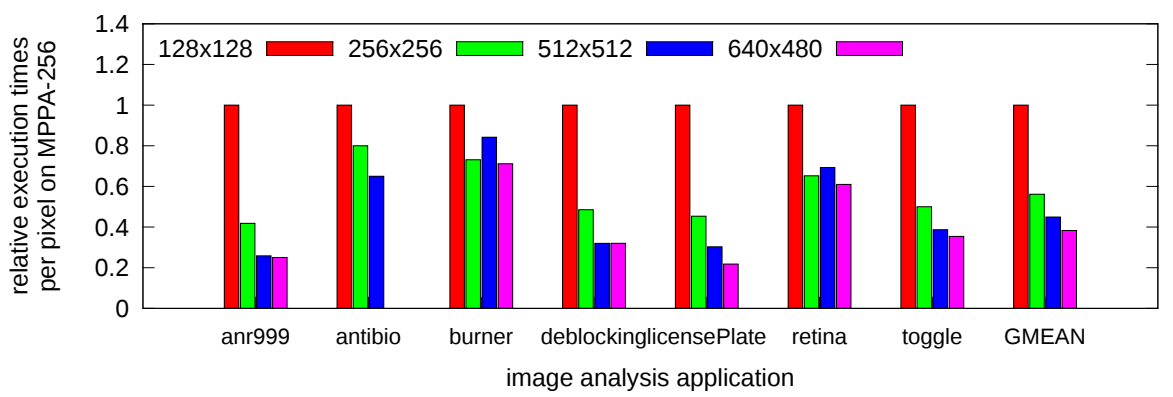

Fig. 9. Impact of the image size on the average execution time per pixel

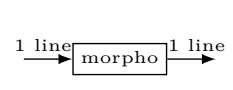

(a) full-image computation

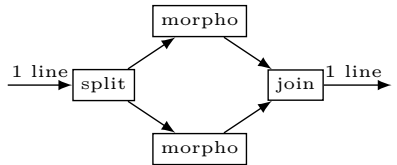

(b) half-line computations

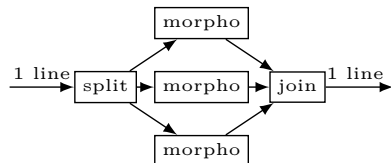

(c) one-third line computations

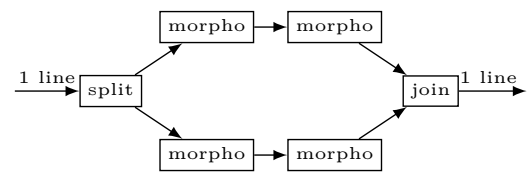

(d) 2 stages half line

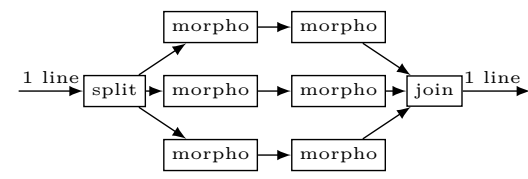

(e) 2 stages one-third line

Fig. 10. Analysed cases of data parallelism for morphological operators

better amortized. This also simplifies the implementation of stencil operators by easing the access to neighboring pixels.

The morphological agents, the most complex operators, have a direct impact on the performance of our applications. Consisting of an aggregate function (min, max, average) on the value of a subset of the neighbor pixels, they are often used in large pipelines in image analysis applications. We used several optimizations during their implementation. Firstly, as stencil operators, they need to access not only the current processed line, but also the previous and the next lines. As a consequence, each agent has a 3-line internal buffer to store the input lines needed for computation. Also, the incoming input lines are stored into this 3lines buffer and processed in a round-robin manner, avoiding time-consuming copies. Finally, these agents benefitted from an optimized assembly kernel to use guarded instructions not automatically generated by the compiler.

We also investigated data parallelism by splitting input lines and computing each portion with several morphologic agents. This approach allows us to take advantage of the MPPA-256 unused compute cores, since application DAGs are usually much smaller than the number of available cores. Because morphological 


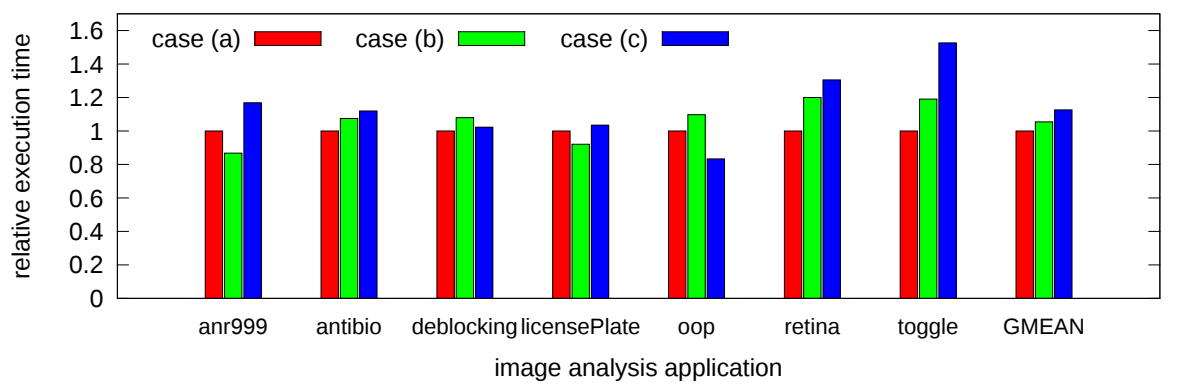

Fig. 11. Execution times with parallel morphological operators

operators are stencils, we use overlapping lines when splitting and joining. Our measures showed that having several stages of computing agents in the (d) and (e) cases of Figure 10 slows down the whole process, so we focused on comparing the (a), (b) and (c) 1-stage cases represented in Figure 10. As shown in Figure 11, the results are quite mixed and application-dependent. Replacing one morphological agent by four or more agents induces more inter-cluster communications, leading to a loss in performance, even if computed data is reduced by half.

\section{2 $\Sigma \mathrm{C}$ code generation}

As stated in Section 2, our compilation chain produces DAGs of elementary image analysis operators from which we generate $\Sigma \mathrm{C}$ subgraphs using our image analysis agents. For example, Figure 12 shows an extract of the generated $\Sigma \mathrm{C}$ code from the FREIA source code in Figure 3.

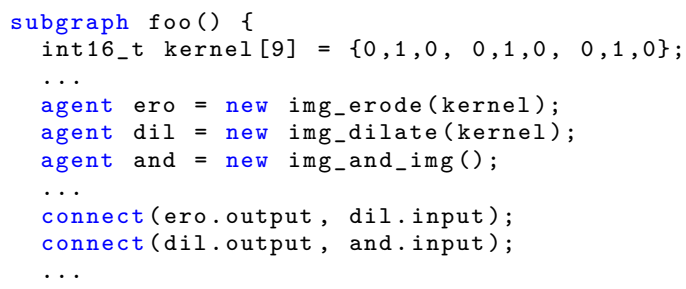

Fig. 12. Corresponding $\Sigma \mathrm{C}$ code to FREIA code in Figure 3

In order to be correctly transposed into a running dataflow program, we must ensure that there is no scalar dependency between two agents of the same subgraph. Indeed, since images are processed on a per line basis, a scalar produced from a whole image cannot be applied on the lines of the same image by an other agent on the same subgraph without causing lines accumulation in inter-agent 
buffers, and thus major performance loss. A split-on-scalar-dependencies pass is used ahead of our $\Sigma \mathrm{C}$ generator to provide scalar-independent DAGs, which can then be transposed directly to $\Sigma \mathrm{C}$ subgraphs.

Some complex image analysis operators involve a convergence loop over an image-dependent parameter. Such operators, being idempotents, can be unrolled with no consequences on the final result. However, this unrolling pass leads to a greater number of generated $\Sigma \mathrm{C}$ agents, and thus an increase occupation of the MPPA compute cores. We measured the influence of the unrolling factor of these particular loops on the execution times of the relevant applications (see Figure 13). Our results show that unrolling dramatically increases the performance of our applications. For these applications, an unrolling factor of 8 leads to a fair speedup while mobilizing a reasonable amount of compute cores.

Split and unrolled image expression DAGs are then encoded as $\Sigma \mathrm{C}$ subgraphs. Our implementation of the generation of $\Sigma \mathrm{C}$ subgraphs is pretty straighforward: for each vertex of one image expression DAG, our compiler PIPS generates an $\Sigma \mathrm{C}$ instantiation statement for the corresponding $\Sigma \mathrm{C}$ agent first, then connection statements between the agent instance and its predecessors or successors in the DAG. Small differences between the input DAGs structure and the $\Sigma \mathrm{C}$ dataflow model have been addressed during this implementation: (1) since the number of inputs and outputs of our $\Sigma \mathrm{C}$ agents are predetermined, we have to insert replication agents when required; (2) DAG inputs and outputs are specific cases and must be dealt with separately; (3) scalar dependencies must be provided to the correct agents by a dedicated path. Similarly, scalar results must be sent back to the host.

In the dataflow model, the slowest task has the greatest impact on the global execution. Since arithmetic operators do little computation compared to morphologic ones, we investigated the fusion of connected arithmetic operators into compound $\Sigma \mathrm{C}$ agents, thus freeing some under-used compute cores. We implemented this pass on top of our $\Sigma \mathrm{C}$ generator. We tested our optimization pass onto several applications with a variable number of merged operators. Execution time results (Figure 14) show little to no difference in performance compared to the reference one agent/operator application. These measures confirm that ag-

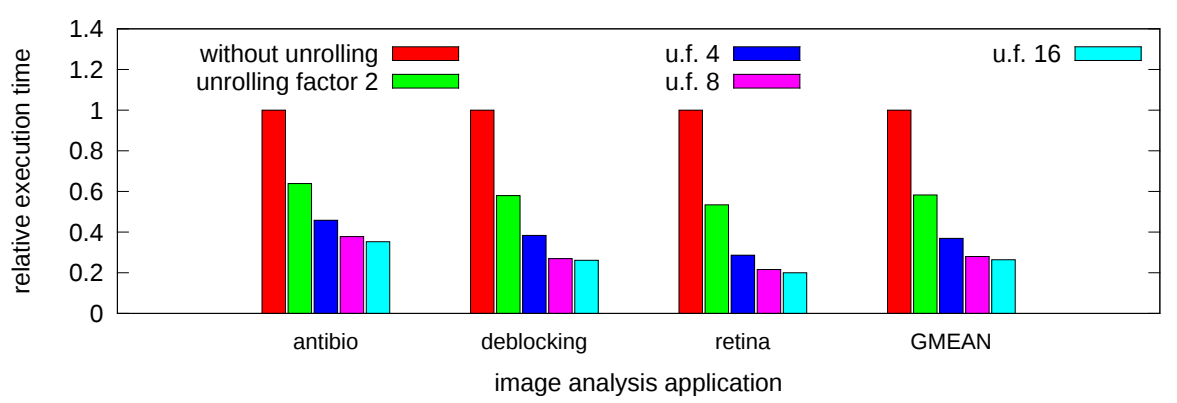

Fig. 13. Relative execution time as a function of unrolling factor 


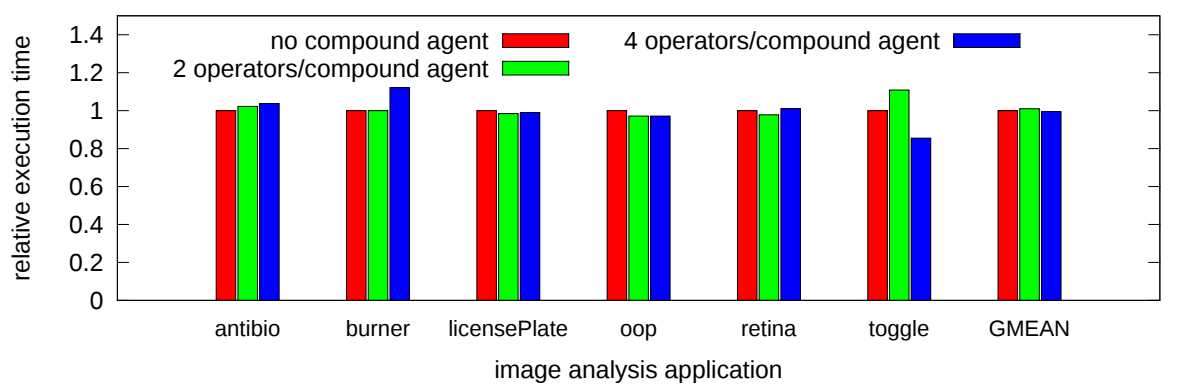

Fig. 14. Influence of the fusion of connected arithmetic operators on execution times

gregated operators are not limiting the global execution while freeing computing power, therefore validating our approach.

\subsection{Runtime environment}

$\Sigma \mathrm{C}$ code generated by our compilation chain often includes several independant and non-connected subgraphs that are all mapped on the MPPA cores. In order to launch the adequate subgraphs at the right time and to control the I/Os, we developed a small runtime in C. It runs on top of the generated $\Sigma \mathrm{C}$ applications and communicates with them through Unix named pipes, as depicted in Figure 2. For each function of this runtime, we added a dedicated $\Sigma \mathrm{C}$ subgraph with agents mapped on the host CPU and the I/O clusters cores to handle the task. This runtime is also used for loading and saving images from the host hard drive. To this end, we use a software implementation of FREIA, called Fulguro [11].

The other dedicated control functions allow us to allocate and free the accelerator embedded RAM, to send or receive images to or from the MPPA, and to launch a compute subgraph onto one or more images. On the $\Sigma \mathrm{C}$ side of the application, several independant subgraphs manage the control signals sent through the named pipes and transfer them to the chip.

The general design of our compilation chain, based on a source code generator, an elementary library and a small runtime environment, allowed us to quickly get functionnal applications on the MPPA-256. With the implementation of a set of specific optimizations (loop unrolling, fusion of fast tasks, splitting of slow tasks, bypassing of the MPPA RAM), we were able to take better advantage of the compute power of this processor. Once this was done, we compared the MPPA-256 to a set of hardware accelerators running the same applications generated by the same compiler.

\section{$5 \quad$ Performance Results}

We have evaluated our compilation chain with eight real image analysis applications covering a wide range of cases. Table 1 shows that our applications contain 


\begin{tabular}{|c|c|c|c|c|c|c|c|}
\hline \multirow{2}{*}{$\begin{array}{l}\text { Apps. } \\
\text { anr999 }\end{array}$} & \multicolumn{3}{|c|}{$\begin{array}{c}\text { \#operators } \\
\text { arith morpho reduct }\end{array}$} & total & \multirow{2}{*}{\begin{tabular}{|c|} 
\#subgraphs \\
1
\end{tabular}} & \multirow{2}{*}{\begin{tabular}{|c|} 
\#clusters \\
2
\end{tabular}} & \multirow{2}{*}{\begin{tabular}{|l} 
image size \\
$224 \times 288$
\end{tabular}} \\
\hline & 0 & 20 & 3 & 23 & & & \\
\hline antibio & 8 & 41 & 25 & 74 & 8 & 6 & $256 \times 256$ \\
\hline bl & 18 & 410 & 3 & 431 & 3 & 16 & $256 \times 256$ \\
\hline deblocking & 23 & 9 & 2 & 34 & 2 & 10 & $512 \times 512$ \\
\hline licensePlate & 4 & 65 & 0 & 69 & 1 & 5 & $640 \times 383$ \\
\hline oop & 7 & 10 & 0 & 17 & 1 & 2 & $350 \times 288$ \\
\hline retina & 15 & 38 & 3 & 56 & 3 & 4 & $256 \times 256$ \\
\hline toggle & 8 & 6 & 1 & 15 & 1 & 1 & $512 \times 512$ \\
\hline
\end{tabular}

Table 1. Characteristics of used image analysis applications

\begin{tabular}{|c|c|c|c|c|c|c|c|c|c|c|}
\hline \multirow{3}{*}{ Apps. } & \multicolumn{2}{|c|}{ Software } & \multicolumn{8}{|c|}{ Hardware } \\
\hline & & & & FPC & GA & & & OpenC & CL & \\
\hline & Flgr & $\mathrm{i} \Sigma \mathrm{C}$ & MPPA & $\mathrm{SPoC}$ & TPX & $\mathrm{i} 2 \mathrm{c}$ & $\mathrm{a} 4 \mathrm{c}$ & GTX & Quadro & Tesla \\
\hline anr999 & 4.3 & 36.5 & 8.3 & 0.9 & 3.5 & 12.2 & 7.2 & 9.8 & 1.5 & 0.9 \\
\hline antibio & 80.2 & 1026 & 670 & 41.9 & 88.5 & 254.1 & 135.3 & 204.3 & 57.4 & 93.4 \\
\hline burner & 795.3 & 814.4 & 113 & 17.2 & 83.8 & 162.2 & 124.4 & 321.0 & 576.2 & 142.0 \\
\hline deblocking & 141.4 & 121.0 & 84 & 30.7 & 11 & 25.1 & 16.7 & 11.1 & 3.5 & 1.3 \\
\hline licensePlate & 483.9 & 152.7 & 20.2 & 13.3 & 36.8 & 32.2 & 21.9 & 36.6 & 7.3 & 2.3 \\
\hline oop & 4.0 & 39.3 & 11.3 & 124.6 & 63.3 & 12.3 & 8.3 & 5.8 & 1.8 & 1.0 \\
\hline retina & 149.0 & 222.5 & 95 & 7.4 & 32.4 & 93.5 & 55.4 & 75.5 & 60.8 & 33.9 \\
\hline toggle & 6.2 & 69.8 & 22.6 & 12.6 & 4.3 & 15.0 & 9.0 & 6.3 & 1.4 & 0.7 \\
\hline$\overline{\mathrm{AVG} / \mathrm{MPPA}}$ & 1.13 & 3.28 & 1.00 & 0.32 & 0.49 & 0.85 & 0.54 & 0.64 & 0.23 & 0.12 \\
\hline
\end{tabular}

Table 2. Execution times (ms) of our applications on the different hardware targets

from 15 elementary operators (toggle) to more than 400 (burner), most of them morphological operators. Table 1 also illustrates the number of $\Sigma \mathrm{C}$ subgraphs generated by our compilation chain, and the number of occupied compute clusters when running on the MPPA-256. These applications generally include less than three independent image expressions DAGs.

Our compilation chain targets several software and hardware accelerators: a reference software implementation, Fulguro [11], on an Intel Core i7-3820 quadcore CPU running at $3.6 \mathrm{GHz}$ with an average power consumption of $130 \mathrm{~W} ; \Sigma \mathrm{C}$ code running directly on the same $\mathrm{CPU}(\mathrm{i} \Sigma \mathrm{C})$; the MPPA-256 processor $(10 \mathrm{~W})$ using $\Sigma \mathrm{C}$; SPoC [13] and Terapix (TPX) [8], two image processing accelerators implemented on a FPGA (26 W); two CPUs using OpenCL [23]: an Intel dualcore (i2c-65 W) and an AMD quad-core CPU (a4c-60W); and three NVIDIA GPUs again with OpenCL [23]: a GeForce 8800 GTX (120 W), a Quadro 600 $(40 \mathrm{~W})$ and a Tesla C $2050(240 \mathrm{~W})$.

We compared the output of our compilation chain from the previously described applications onto these 8 hardware targets, both in terms of execution times and energy consumption. For the MPPA-256 chip, time and energy measures were obtained using Kalray's k1-power utility software, ignoring transfers and control CPU consumption. Time figures for the FPGAs, CPUs and GPUs 


\begin{tabular}{|c|c|c|c|c|c|c|c|c|c|c|c|}
\hline \multirow{3}{*}{ Apps. } & \multicolumn{2}{|c|}{ Software } & \multicolumn{9}{|c|}{ Hardware } \\
\hline & & & MP & PA & $\mathrm{FPC}$ & GA & & & OpenC & $\mathrm{CL}$ & \\
\hline & Flgr & $\mathrm{i} \Sigma \mathrm{C}$ & real & ideal & SPoC & TPX & i2c & $\mathrm{a} 4 \mathrm{c}$ & |GTX| & Quadro & Tesla \\
\hline anr999 & 559 & 4745 & 50 & 6 & 23 & 91 & 793 & 432 & 1176 & 60 & 221 \\
\hline anti & 10425 & 133380 & 3500 & 1313 & 1089 & 2301 & 16517 & 8118 & 24516 & 2296 & 22883 \\
\hline burner & 103390 & 105900 & 388 & 388 & 447 & 2179 & 10543 & 7464 & 38520 & 23048 & 34790 \\
\hline deblocking & 18382 & 15730 & 431 & 269 & 798 & 286 & 1632 & 1002 & 1332 & 140 & 319 \\
\hline licensePlate & 62907 & 24284 & 120 & 38 & 354 & 957 & 2093 & 1314 & 4392 & 292 & 564 \\
\hline oop & 520 & 5110 & 59 & 7 & 3240 & 1646 & 800 & 498 & 696 & 72 & 245 \\
\hline retina & 19370 & 28925 & 487 & 122 & 192 & 842 & 6078 & 3324 & 9060 & 2432 & 8306 \\
\hline toggle & 806 & 9074 & 119 & 7 & 328 & 112 & 975 & 540 & 756 & 56 & 172 \\
\hline AVG/MPPA & 28.78 & 83.38 & 1.00 & 0.25 & 1.65 & 2.52 & 10.81 & 6.33 & 15.01 & 1.79 & 5.45 \\
\hline
\end{tabular}

Table 3. Energy $(\mathrm{mJ})$ used by our test cases on different targets

were taken from [14]. Energy figures were derived from target power consumption. Results are shown in Table 2 and Table 3, with best performances in bold. Compared to the Fulguro monothreaded sofware implementation, $\Sigma \mathrm{C}$ on CPU is relatively slow, due to the numerous threads communicating with each other. To take individual MPPA-256 compute cluster power supply into account, we added in Table 3 a column "MPPA ideal" representing the energy of clusters actually used for the computations, according to Table 1 . Disconnecting unused compute cores would provide us an extra $\times 4$ speedup.

These results show that although MPPA $/ \Sigma \mathrm{C}$ is not faster than dedicated hardware targets, it provides the lowest average energy consumption for tested applications. The high degree of task parallelism induced by the use of the $\Sigma \mathrm{C}$ dataflow language on the 256 cores of the MPPA-256 processor is thus a strength facing dedicated hardware in low energy and embedded applications.

\section{Conclusion and Future Work}

We added a new hardware target to the FREIA ANR project: a 256 cores processor with a power consumption of $10 \mathrm{~W}$ through the use of the $\Sigma \mathrm{C}$ dataflow programming language. Using the PIPS source-to-source compiler, we generated $\Sigma \mathrm{C}$ dataflow code based upon a small image analysis library written in $\Sigma \mathrm{C}$. The execution of the generated applications relies on a small runtime environment controlling the execution of the different $\Sigma \mathrm{C}$ subgraphs mapped on the cores of the MPPA-256 processor. We implemented a set of specific optimizations from automatic fast operator aggregation to data-parallel slow operators to achieve better performance. The performance of our approach is shown by comparing the MPPA-256 results to other hardware accelerators using the same compilation chain. MPPA $/ \Sigma \mathrm{C}$ proves to be the most energy-efficient programmable target, which competes in performance with specific image-processing dedicated hardware such as the SPoC FPGA processor.

In the current approach, several subgraphs are mapped onto different compute cores, meaning only a fraction of the chip is used at a given time. Future 
work includes the investigation of dynamically mapping distinct $\Sigma \mathrm{C}$ subgraphs on the same cores when they do not need to be run concurrently. Another way to save energy, especially for small applications, would be the ability to disconnect unused clusters within the chip, as shown in column "MPPA ideal" in Table 3. More performance improvements could also be obtained on some applications by generating automatically kernel-specific convolutions, which would reduce execution time by skipping altogether null-weighted pixels.

Aknowledgements Thanks to Danielle Bolan and Pierre Jouvelot for proofreading, Antoniu Pop for his advises and bibliography pointers, Kalray engineers Frédéric Blanc, Jérôme Bussery and Stéphane Gailhard for their support and to anonymous reviewers whose comments greatly helped to improve this paper.

\section{References}

1. The StreamIt Language (2002), http://www.cag.lcs.mit.edu/streamit/

2. Tera-scale architecture (2008), https://www-asim.lip6.fr/trac/tsar/wiki

3. The TilePro64 many-core architecture (2008), http://www.tilera.com/

4. The Epiphany many-core architecture (2012), http://www.adapteva.com/

5. Aubry, P., Beaucamps, P.E., Blanc, F., Bodin, B., Carpov, S., Cudennec, L., David, V., Dore, P., Dubrulle, P., Dupont de Dinechin, B., Galea, F., Goubier, T., Harrand, M., Jones, S., Lesage, J.D., Louise, S., Chaisemartin, N.M., Nguyen, T.H., Raynaud, X., Sirdey, R.: Extended Cyclostatic Dataflow Program Compilation and Execution for an Integrated Manycore Processor. In: Alexandrov, V.N., Lees, M., Krzhizhanovskaya, V.V., Dongarra, J., Sloot, P.M.A. (eds.) ICCS. Procedia Computer Science, vol. 18, pp. 1624-1633. Elsevier (2013)

6. Bandishti, V., Pananilath, I., Bondhugula, U.: Tiling stencil computations to maximize parallelism (Nov 2012)

7. Bilodeau, M., Clienti, C., Coelho, F., Guelton, S., Irigoin, F., Keryell, R., Lemonnier, F.: FREIA: FRamework for Embedded Image Applications (2008-2011), freia.enstb.org, French ANR-funded project with ARMINES (CMM, CRI), THALES (TRT) and Télécom Bretagne

8. Bonnot, P., Lemonnier, F., Edelin, G., Gaillat, G., Ruch, O., Gauget, P.: Definition and SIMD Implementation of a Multi-Processing Architecture Approach on FPGA. In: Design Automation and Test in Europe. pp. 610-615. IEEE (Dec 2008)

9. Bosilca, G., Bouteiller, A., Danalis, A., Herault, T., Lemarinier, P., Dongarra, J.: DAGuE: A generic distributed DAG engine for high performance computing. Parallel Computing 38(1-2), 37-51 (Jan 2012), http://linkinghub.elsevier.com/ retrieve/pii/S0167819111001347

10. Chambers, C., Raniwala, A., Perry, F., Adams, S., Henry, R.R., Bradshaw, R., Weizenbaum, N.: FlumeJava: easy, efficient data-parallel pipelines. p. 363. ACM Press (2010), http://portal.acm.org/citation.cfm?doid=1806596.1806638

11. Clienti, C.: Fulguro image processing library. Source Forge (2008)

12. Clienti, C.: Architectures flots de données dédiées au traitement d'images par la Morphologie MATHÉMATIQUE. Ph.D. thesis, MINES ParisTech (Sep 2009)

13. Clienti, C., Beucher, S., Bilodeau, M.: A system on chip dedicated to pipeline neighborhood processing for mathematical morphology. In: EUSIPCO: European Signal Processing Conference (Aug 2008) 
14. Coelho, F., Irigoin, F.: API compilation for image hardware accelerators. ACM Transactions on Architecture and Code Optimization 9(4), 1-25 (Jan 2013)

15. CRI, MINES ParisTech: PIPS (1989-2012), pips4u.org, open Source Research Compiler, under GPLv3

16. Datta, K., Murphy, M., Volkov, V., Williams, S., Carter, J., Oliker, L., Patterson, D., Shalf, J., Yelick, K.: Stencil computation optimization and auto-tuning on stateof-the-art multicore architectures. In: SC'08: Conference on Supercomputing. pp. 1-12. IEEE Press (2008)

17. Dupont de Dinechin, B., Sirdey, R., Goubier, T.: Extended cyclostatic dataflow program compilation and execution for an integrated manycore processor. In: Procedia Computer Science 18 (2013)

18. Gordon, M.I., Thies, W., Amarasinghe, S.: Exploiting coarse-grained task, data, and pipeline parallelism in stream programs. ACM SIGPLAN Notices 41(11), 151 (Oct 2006), http://portal. acm.org/citation. cfm?doid=1168918.1168877

19. Goubier, T., Sirdey, R., Louise, S., David, V.: $\Sigma$ C: a programming model and language for embedded manycores (2011)

20. Halbwachs, N., Caspi, P., Raymond, P., Pilaud, D.: The synchronous dataflow programming language LUSTRE. In: Proceedings of the IEEE. pp. 1305-1320 (1991)

21. Irigoin, F., Jouvelot, P., Triolet, R.: Semantical interprocedural parallelization: an overview of the PIPS project. In: Proceedings of ICS 1991. pp. 244-251. ACM Press (1991)

22. Kahn, G.: The semantics of a simple language for parallel programming. p. 5 (1974)

23. KHRONOS Group: OpenCL Computing Language v1.0 (Dec 2008)

24. Le Guernic, P., Benveniste, A., Bournai, P., Gautier, T.: Signal-A data floworiented language for signal processing. Acoustics, Speech and Signal Processing, IEEE Transactions on 34(2), 362-374 (Apr 1986)

25. Lee, E.A., Messerschmitt, D.G.: Static Scheduling of Synchronous Data Flow Programs for Digital Signal Processing. IEEE Trans. Comput. 36(1), 24-35 (Jan 1987)

26. Murthy, P.K., Lee, E.A.: Multidimensional synchronous dataflow. IEEE Transactions on Signal Processing 50, 3306-3309 (2002)

27. OpenMP Architecture Review Board: OpenMP Application Program Interface, Version 3.0 (May 2008)

28. Pop, A.: Leveraging Streaming for Deterministic Parallelization - an Integrated Language, Compiler and Runtime Approach. Ph.D. thesis, MINES ParisTech (Sep 2011)

29. Ragan-Kelley, J., Barnes, C., Adams, A., Paris, S., Durand, F., Amarasinghe, S.: Halide: A language and compiler for optimizing parallelism, locality, and recomputation in image processing pipelines. PLDI 2013 p. 12 (Jun 2013)

30. Soile, P.: Morphological Image Analysis: Principles and Applications. SpringerVerlag (2003)

31. Stephens, R.: A Survey Of Stream Processing (1995) 